\title{
Uncertainty Analysis in Prestack Stratigraphic Inversion: a first insight
}

\section{Authors}

Frédéric Delbos (IFP), Kaoutar Sghiouer (Université Technologique de Compiègne, anciennement IFP), Delphine Sinoquet (IFP).

\begin{abstract}
Stratigraphic inversion of prestack seismic data allows the determination of subsurface elastic parameters (density, $\mathrm{P}$ and S-impedances). Based on a Bayesian approach, the problem is formulated as a non-linear least-squares local optimization problem. The objective function to be minimized is composed of two terms, the first one measures the mismatch between the synthetic seismic data (computed via a forward operator) and the observed seismic data, the second one models geological a priori information on the subsurface model.

It is crucial to estimate the a posteriori uncertainties because the solution model of the inversion is only one solution among the range of admissible models that fit the data and the a priori information. The goal of this paper is to propose an optimized deterministic method to estimate a posteriori uncertainties in stratigraphic inversion.

The proposed method is based on the hypothesis that the covariance matrices describing the uncertainties on the data and on the model are laterally uncorrelated (no cross correlation among parameters of different traces). Moreover, the covariance matrix on the data is also supposed laterally stationary. Application on 2D synthetic PP data illustrates the performances of the method. Extension and limitations of the method are discussed.
\end{abstract}




\section{Introduction}

In a Bayesian approach, which is a natural formulation for many geophysical problems, solving an inverse problem consist not only in estimating the best probable set of subsurface parameters but to estimate the posterior probability density function (PDF) on the model space. This is particularly true in seismic facies analyses of 4D data where the noise level is in the magnitude of interpreted parameters : from on vintage to the other very small differences in subsurface elastic parameters are correlated a posteriori to production effects (see Nivlet 2007). Presently, numerical methods for estimation of posterior PDF can be gathered into at least two classes: the class of stochastic approaches which is mainly formed of Markov Chain Monte Carlo method, and the class of deterministic approaches which includes linearized a posteriori analysis. Often actual methods combine elements of the two classes, but their main features make them belonging to one of them.

In stochastic approaches, no assumption on the posterior PDF function is needed. The Monte Carlo method consists in sampling the prior PDF function in the model space and then uses a Metropolis type algorithm with a random walk to map the posterior PDF function (see Mosegaard 1998 for example). Considering the large size of the model and data space (up to $10^{8}$ unknowns and data samples in stratigraphic inversion) and the computational cost of the forward modelling, this class of method is often rejected.

Deterministic approaches are based on a gaussian assumption of the posterior PDF and also of all the 'input' probabilities densities. This assumption provides an explicit analytical form of the posterior distribution which can be computed at a lower computational cost (see Tarantola 2005). The computation of the posterior distribution has been the subject of many algorithm studies, we mention the following techniques: frequency methods using Fast Fourier Transform (FFT), and direct methods using factorisation techniques (LU factorisation for example).

In frequency methods, one solves a sequence of small decoupled linear equations obtained by transforming a linearized inversion problem into the Fourier domain (see for instance Buland et al. 2003). Thanks to FFT, this method has the advantage to be very cheap in computational cost. Its drawback comes from the restrictive assumptions to be postulated: the covariance function of the prior model has to be stationary and homogeneous (which not allows applications to complex media with high lateral variations or with more than one geological unit), the ratio between the $\mathrm{P}$-wave velocity and the $\mathrm{S}$-wave velocity is assumed to be constant (which is not the case in general) and finally this method works on a linearized AVO inversion problem (with the loss of the complexity of wave propagation).

Direct methods do not need to work on a linearized version of the inversion problem. Then in a non linear context, uncertainty analysis consists in assuming a gaussian approximation in the vicinity of the optimal subsurface model which has been previously estimated via a non linear optimization method (see Gouveia et al. 1998). Then this approach is less restrictive than the frequency one. Nevertheless, the estimation of the posterior distribution is a more difficult task since it requires the inversion of a very large matrix (its size is $n \times n$ where $n$ is the number of unknowns).

In the following, we first briefly describe an optimized method of posterior uncertainty analysis, based on the deterministic approach coupled with a direct. Then the method is applied on a 2D synthetic seismic data set corresponding to 2D realistic reservoir zones where optimal model and its error bars are plotted.

\section{Uncertainty Analysis for Prestack Stratigraphic Inversion}

For uncertainty analysis we use the prestack stratigraphic inversion algorithm described by Tonellot et al. 2001 which minimizes the objective function

$$
\mathrm{J}(\mathrm{m})=\sum_{\theta}\left\|\mathrm{R}_{\theta}(\mathrm{m}) * \mathrm{w}_{\theta}-\mathrm{d}_{\theta}^{\text {obs }}\right\|_{\mathrm{C}_{\mathrm{d}}^{-1}}^{2}+\left\|\mathrm{m}-\mathrm{m}_{\text {prior }}\right\|_{\mathrm{C}_{\mathrm{m}}^{-1}}^{2}=\mathrm{J}_{\mathrm{S}}(\mathrm{m})+\mathrm{J}_{\mathrm{G}}(\mathrm{m})
$$


composed of two terms. $J_{S}(m)$ is the seismic term where $R_{\theta}(m)$ is the Aki-Richards reflectivity series corresponding to the current model $m$ and to the angle $\Theta, w_{\ominus}$ is the wavelet, and $\mathrm{d}^{\text {obs }}{ }_{\theta}$ is the observed seismic trace. $\mathrm{J}_{\mathrm{G}}(\mathrm{m})$ is the geologic term where $\mathrm{m}_{\text {prior }}$ is the elastic a priori model. $\mathrm{C}_{\mathrm{d}}$ and $\mathrm{C}_{\mathrm{m}}$ are the prior covariance matrices describing uncertainties on the data and on the model, they are assumed here laterally uncorrelated, $\mathrm{C}_{\mathrm{d}}$ is also assumed laterally stationary. The maximum a posteriori (MAP) model is the model $\mathrm{m}_{\text {post }}$ which minimizes $\mathrm{J}(\mathrm{m})$. Then, by a linearization of $\mathrm{R}_{\Theta}(\mathrm{m})$ operator, a gaussian approximation of the posterior PDF centered on $\mathrm{m}_{\text {post }}$ is obtained. This distribution is then quantified by the posterior covariance matrix

$$
\mathrm{C}_{\text {post }}=\left[\sum_{\theta} \mathrm{G}^{\mathrm{T}} \mathrm{W}_{\theta} \mathrm{C}_{\mathrm{d}}^{-1} \mathrm{~W}_{\theta}^{\mathrm{T}} \mathrm{G}+\mathrm{C}_{\mathrm{m}}^{-1}\right]^{-1}=\left[\mathrm{H}_{\mathrm{S}}+\mathrm{C}_{\mathrm{m}}^{-1}\right]^{-1},
$$

where $G$ is the jacobian matrix of $R_{\Theta}(m)$ in $m_{\text {post }}$ and $W_{\Theta}$ is the convolution matrix of the wavelet at angle $\Theta$. It is straightforward to show that the first term $\mathrm{H}_{\mathrm{S}}$ is a Gauss-Newton approximation of the hessian of $\mathrm{J}_{\mathrm{S}}$ in $\mathrm{m}_{\text {post }}$. The second term, which is the inverse of the covariance prior matrix on the model is exactly the hessian of $\mathrm{J}_{\mathrm{G}}$. As the prior covariance matrices are diagonal, $C_{\text {post }}$ is a $n_{T} \times n_{T}$ block diagonal matrix (the parameters are laterally uncorrelated) where $\mathrm{n}_{\mathrm{T}}$ is the number of traces . Then computing $\mathrm{C}_{\text {post }}$ requires two steps:

(1) the computation of the matrix $\mathrm{H}_{\mathrm{S}}$, and

(2) the inversion of the $\mathrm{n}_{T}$ diagonal blocks of $\mathrm{C}_{\text {post }}$.

The first step can be very high in computational cost if not made properly. We have optimized it using two improvements. The first one is to store in a matrix $Z_{\Theta}$ the result of the matrix products $\mathrm{W}_{\Theta} \mathrm{C}^{-1}{ }_{\mathrm{d}} \mathrm{W}^{\mathrm{T}}{ }_{\theta}$. The second one is to use the following explicit analytical formula

$$
\begin{aligned}
& \frac{\partial\left(R_{\theta}\right)_{t 1-1}\left(m_{x, y}\right)}{\partial m_{t 1, p 1, x, y}} \frac{\partial\left(R_{\theta}\right)_{t 2-1}\left(m_{x, y}\right)}{\partial m_{t 2, p 2, x, y}}\left(\widetilde{\omega}_{\theta}^{t 1-1} \otimes \omega_{\theta}\right)_{t 1-t 2}+\frac{\partial\left(R_{\theta}\right)_{t 1-1}\left(m_{x, y}\right)}{\partial m_{t 1, p 1, x, y}} \frac{\partial\left(R_{\theta}\right)_{t 2}\left(m_{x, y}\right)}{\partial m_{t 2, p 2, x, y}}\left(\widetilde{\omega}_{\theta}^{t-1-1} \otimes \omega_{\theta}\right)_{t 1-t 2-1} \\
& +\frac{\partial\left(R_{\theta}\right)_{t 1}\left(m_{x, y}\right)}{\partial m_{t 1, p 1, x, y}} \frac{\partial\left(R_{\theta}\right)_{t 2-1}\left(m_{x, y}\right)}{\partial m_{t 2, p 2, x, y}}\left(\widetilde{\omega}_{\theta}^{t 1} \otimes \omega_{\theta}\right)_{t 1-t 2+1}+\frac{\partial\left(R_{\theta}\right)_{t 1}\left(m_{x, y}\right)}{\partial m_{t 1, p 1, x, y}} \frac{\partial\left(R_{\theta}\right)_{t 2}\left(m_{x, y}\right)}{\partial m_{t 2, p 2, x, y}}\left(\widetilde{\omega}_{\theta}^{t 1} \otimes \omega_{\theta}\right)_{t 1-t 2}
\end{aligned}
$$

which gives the value of the element $((t 1, p 1, x, y),(t 1, p 1, x, y))$ of the matrix $H_{S}(\Theta)$. The 4 uplet $(\mathrm{t}, \mathrm{p}, \mathrm{x}, \mathrm{y})$ is a parameter coordinates in the subsurface grid ( $\mathrm{t}$ is the time index, $\mathrm{p}$ the parameter type index, $\mathrm{x}$ and $\mathrm{y}$ the crossline and inline indices). $\widetilde{\omega}_{\theta}^{t}$ is the $\mathrm{t}^{\text {th }}$ column of the matrix product $\mathrm{C}^{-1} \mathrm{dW}_{\mathrm{d}}^{\mathrm{T}}$ and $\otimes$ is the cross-correlation product. As the cross-correlation values $\left(\tilde{\omega}_{\theta}^{t} \otimes \omega_{\theta}\right)_{i}=\left(Z_{\theta}\right)_{-i, t}$ have been previously stored, the computational cost of $\mathrm{H}_{\mathrm{S}}$ is then optimized (one no need to recompute these values from one trace to the other).

The second step (the inversion of the matrix $\mathrm{C}_{\text {post }}$ ) is more conventional: it can be processed very fast using direct method such as a LU factorisation (see Anderson 1999). In fact, the size of each diagonal block is small (around $1000 \times 1000$ ) and this step is naturally parallelisable (each matrix inversion being send to one processor).

A typical diagonal block of the matrix $\mathrm{H}_{\mathrm{S}}$ is shown in Figure 1. This $3 \times 3$ block matrix shows the autocorrelation of the P-wave impedance, $S$-wave impedance, and density on its main diagonal and the cross-correlation among different parameters in the offdiagonal blocks.

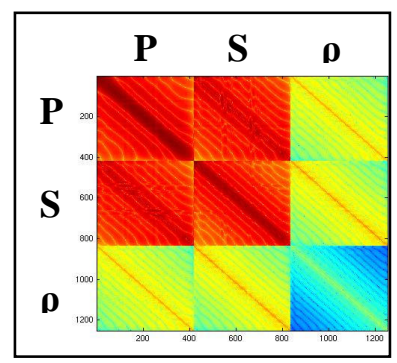

\section{D synthetic study}

The exact model (see raw 1 of Fig. 3 for the elastic parameters) is a 2D crossline of a 3D realistic model representative of a turbiditic complex channel in a deep offshore environment (see Bourgeois et al. 2005). The inversion window is $3 \mathrm{~km}$ long and $960 \mathrm{~m}$ thick $(832 \mathrm{~ms}$ ) from $1.5 \mathrm{~km}$ to $2.46 \mathrm{~km}$ in depth (time range is $600-1432 \mathrm{~ms}$ ). This area is discretized on regular grid of 151 traces (one each $20 \mathrm{~m}$ ) and of 417 time samples (one each $2 \mathrm{~ms}$ ). Using the exact model, the P-P Aki-Richards reflectivity formula and a 1D convolution operator, a seismic data set is generated. Four angle-limited stacks are generated: $05^{\circ}-15^{\circ}$ (see Fig. 2 ), $15^{\circ}-25^{\circ}, 25^{\circ}$ - 
$35^{\circ}, 35^{\circ}-45^{\circ}$. The wavelet used for each angle is a $5-10-60-80 \mathrm{~Hz}$ linear band-pass filter. The prior model (not displayed here) is the exact model filtered using a $20-40 \mathrm{~Hz}$ low-pass filter. The prior covariance matrices $\mathrm{C}_{\mathrm{d}}$ and $\mathrm{C}_{\mathrm{m}}$ are choosen diagonal and respectively filled with a $1 \%$ noise-to-signal ratio and a $5 \%$ standard deviation with respect to the average values of exact elastic parameters: $\sigma_{\text {prior }}^{\mathrm{P}}=465, \quad \sigma_{\text {prior }}^{\mathrm{S}}=270 \quad$ and $\sigma_{\text {prior }}^{\rho}=0.12$.



Figure 2: (near section) synthetic seismic (left); residuals (right)

Then, the MAP model $m_{\text {post }}$ (see raw 2 of Fig. 3) and its associated residuals (see Fig. 2) are computed through the inversion process. The estimated elastic parameters are well recovered : $\mathrm{m}_{\text {post }}$ is very close from $\mathrm{m}_{\text {exact }}$ and the residuals are almost null (noiseless seismic data). Using the method described previously an uncertainty analysis is processed : absolute $(\sigma)$ and relative $\left(\sigma_{\text {rel }}: \sigma\right.$ normalysed by $\left.\sigma_{\text {prior }}\right)$ standard deviations for $m_{\text {post }}$ are respectively displayed in raw 3 and 4 of Figure 2. That provides insight into the different degree of data resolution for the 3 elastic parameters. The prior standard deviations are reduced to the ranges of : 40-100 (8\%-20\%) for the P-impedance parameters, 44-80 (16\%-30\%) for the S-impedance and 0.066-0.08 (54\%-67\%) for the density. It confirms the already known fact that for P-P data the P-impedance is the best resolved parameter, followed by the S-impedance and then the density : left and right parts of Figure 4 respectively shows that the P-impedance is 1.2 to 1.3 times better resolved than $\mathrm{S}$-impedance and 3 to 5.5 times better resolved than the density. Note that, this analysis is neglecting the off-diagonal terms of the posterior covariance matrix. A more detailed analysis would consist in generating pseudo-random realizations of posterior subsurface models. This analysis is straightforward once $\mathrm{C}_{\text {post }}$ has been evaluated.

\section{Conclusions}

The proposed method, optimized for laterally uncorrelated prior matrices and a laterally stationnary prior matrix on the data, gives a first insight towards a complete uncertainty analysis. Generalization to multicomponent (see DeVault et al. 2007) or 4D prestack joint inversion is in principle straightforward. The stated assumptions may be restrictive. Nethertheless, for some realistic case, where for example the horizontal correlation length is small with respect to the horizontal dimensions of the model, choosing diagonal prior matrices may be appropriate. Moreover, a patent is being written on the extension of this method using exponential covariance operator on the prior model.

\section{References}

Anderson, E. et al [1999] Lapack user Guide, Third Edition. SIAM Publication.

Bourgeois, A., Labat, K., Euzen, T., Froideveau, P., Le Bras, C. [2005], How to build a geological and geophysical realistic synthetic seismic data set for benchmarking reservoir studies?. $75^{\text {th }}$ Annual International Meeting, Expended Abstract, SEG, 1378-1382.

Buland, A., Kolbjørnsen,O., and Omre, H. [2003] Rapid spatially coupled AVO inversion in the Fourier domain. Geophysics, 68, NO. 3, 824-836.

DeVault, B., Tonnellot, T., Macé, D., Ker, S. and Pichard, M. [2007] Prestack 9-C joint inversion for stratigraphic prediction in the Williston Basin. $78^{\text {st }}$ Annual International Meeting, Expended Abstract, SEG, 1039-1043.

Gouveia, W. P., and Scales, J. A. [1998] Bayesian seismic waveform inversion: Parameter estimation and uncertainty analysis. J. Geophys. Res., 103, 2759-2779.

Mosegaard, K. [1998] Resolution analysis of general inverse problems through inverse Monte Carlo Sampling. Inverse Problems, 14, 405-426. 


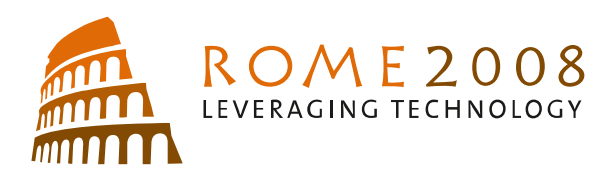

Nivlet, P., Tonellot, T., Sexton. P., Piazza, J. L., Lefeuvre, F., and Duplantier, O. [2006] Building 4D Constraint for History Matching from Stratigraphic Pre-Stack inversion: Application to the Girassol Field. EAGE $68^{\text {th }}$ Conference \& Exhibition.

Nivlet, P. [2007] Uncertainties in Seismic Facies Analysis for Reservoir Characterisation or Monitoring : Causes and Consequences. Oil \& Gas Sci. and tech., 62, No. 2, 225-235.

Tarantola, A. [2005] Inverse Problem Theory. SIAM Publication.

Tonellot, T., Macé, D., and Richard, V. [2001] Joint stratigraphic inversion of angle-limited stacks. $71^{\text {st }}$ Annual International Meeting, Expended Abstract, SEG, 227-230.

\section{Acknowledgments}

We would like to thank Patrick Lailly and Thierry Tonellot for helpful discussions. We acknowledge Karine Labat, Karine Broto and Vincent Clochard for their precious help for the application.

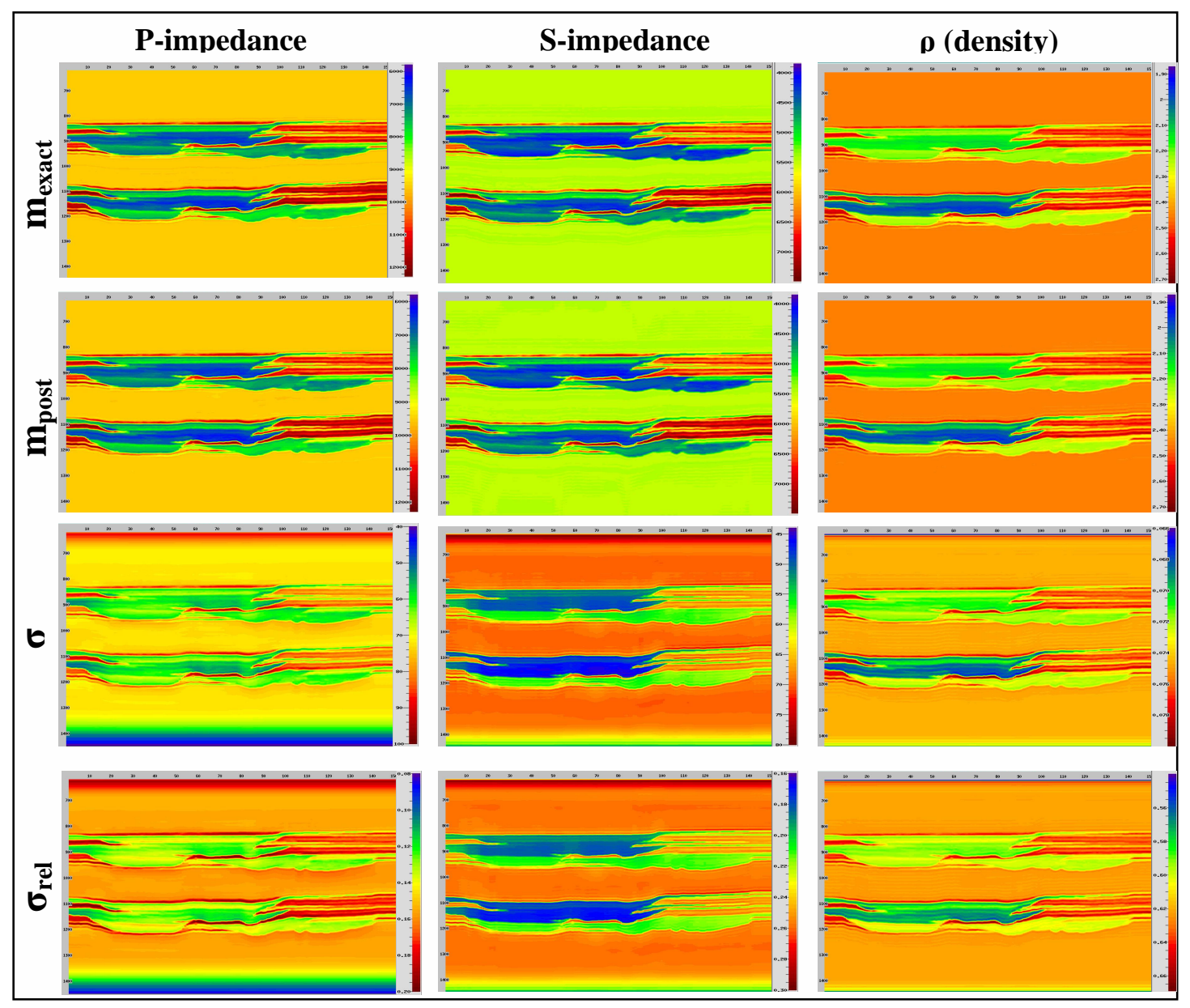

Figure 3: result table of inversion and uncertainty analysis

The result type is in row and the parameter type is in column
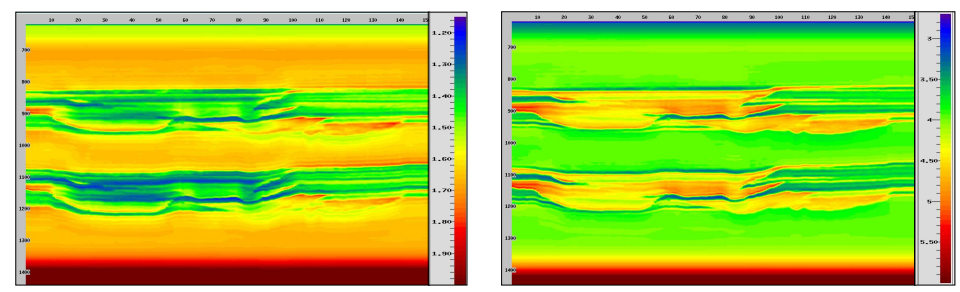

Figure 4: $\sigma_{\text {rel }}^{\mathrm{P}}$ over $\sigma_{\text {rel }}^{\mathrm{S}}($ left $) ; \sigma_{\text {rel }}^{\mathrm{P}}$ over $\sigma_{\text {rel }}^{\mathrm{\rho}}$ (right) 